\title{
Características generales, propiedades, yacimientos y aplicaciones de pirofilita. \\ II. Yacimientos, aplicaciones y utilización como materia prima cerámica
}

\author{
P.J. SÁNCHEZ SOTO y J.L. PÉREZ RODRÍGUEZ \\ Instituto de Ciencia de Materiales de Sevilla, Centro Mixto Consejo Superior de Investigaciones Científicas (C.S.I.C.) \\ Universidad de Sevilla, Isla de la Cartuja, 41092-Sevilla.
}

En este trabajo se indican los principales yacimientos mundiales de pirofilita según la bibliografía, los datos de producción disponibles, además de revisar las investigaciones más destacadas sobre prospección y caracterización de materias primas que contenienen pirofilita y que presentan un marcado interés aplicado. Se realiza una amplia revisión de las principales aplicaciones a las que se destinan dichas materias primas, en especial para la preparación de materiales cerámicos y refractarios, su uso como carga, en la construcción, obtención de catalizadores y síntesis de materiales a alta presión debido a sus propiedades.

Por último, se describen y revisan sus aplicaciones en la preparación de nuevos materiales y materiales avanzados, como son los sialones, destacando su potencial de utilización en nuestro país como resultado de diversas investigaciones de interés, realizadas por distintos Institutos y Departamentos universitarios. Se deduce que la pirofilita adquiere una importancia relativa cada vez mayor en relación a otros silicatos laminares, como son talco y caolinita.

Palabras clave:Pirofilita, politipismo, deshidroxilación, mullita, refractarios, alta presión, materiales avanzados.

Pyrophyllita. General Properties. Part II: Deposits, applications and uses as ceramic raw material.

In this work, the main world deposits of pyrophyllite are indicated according to the literature, showing the available production data. It is emphasized the research focusing on mining and characterization of raw materials containing pyrophyllite, which shows a marked applied interest. It is described and reviewed the most relevant applications of pyrophyllite and pyrophyllite raw materials, in especial refractories and ceramics, fillers, building materials, catalysts and as a high-pressure medium for synthesis of materials.

Finally, it is also described and reviewed its use as raw material for the preparation of advanced materials, such as sialon, emphasizing the possibilities of application of pyrophyllite raw materials in our country as a result of distinct investigations of relevant interest performed by several Research Institutes and University Departments. It is deduced that pyrophyllite and pyrophyllite raw materials are increasing in relative importance in many applied fields, as compared with other layer silicates, such as talc and kaolinite.

Keywords: Pyrophyllite, polytypism, dehydroxylation, mullite, refractories, high-pressure, advanced materials.

\section{INTRODUCCION}

En la primera parte de la presente revisión, se han expuesto y descrito las investigaciones realizadas sobre la estructura de la pirofilita, sus propiedades, su síntesis en el laboratorio y la formación de soluciones sólidas. Se describieron, revisaron y discutieron las investigaciones realizadas sobre sus características propiedades térmicas, en concreto los procesos de deshidroxilación y formación de fases de alta temperatura, de mayor interés para sus aplicaciones como materia prima cerámica, así como su rehidroxilación. Asimismo, se prestó atención a las investigaciones realizadas sobre la formación de mullita a partir de pirofilita y materias primas que la contienen mediante tratamientos mecánicos y térmicos, además de su alta inercia química en medio ácido fuerte que permite incluso separarla de otros silicatos presentes en mezclas naturales con pirofilita.

En esta segunda parte, se indican los principales yacimien- tos mundiales de pirofilita según la bibliografía y se dan los datos de producción disponibles. Se revisan las investigaciones más destacadas sobre prospección y caracterización de materias primas que contenienen pirofilita y que presentan un marcado interés aplicado. Por otra parte, se realiza una amplia revisión de las principales aplicaciones a las que se destinan dichas materias primas, en especial para la preparación de materiales cerámicos y refractarios, su uso como carga, en la construcción, obtención de catalizadores y síntesis de materiales a alta presión debido a las propiedades de la pirofilita.

Por último, se describen y revisan las aplicaciones de la pirofilita en la preparación de nuevos materiales y materiales avanzados, como son los sialones, destacando su potencial de utilización en nuestro país como resultado de diversas investigaciones de interés, realizadas por distintos Institutos y Departamentos universitarios. 


\section{YACIMIENTOS Y PRODUCCION DE PIROFILITA Y MATERIAS PRIMAS QUE LA CONTIENEN}

\subsection{Yacimientos mundiales de interés}

Hasta hace relativamente pocos años, los datos de producción de pirofilita y talco se presentaban conjuntos, puesto que al ser ambos minerales tan similares en propiedades, algunos yacimientos supuestamente explotados como talco, en realidad eran de pirofilita. Sin embargo, en círculos comerciales se contempla ya la pirofilita como un mineral con una importancia propia, sobre todo por sus aplicaciones en la industria cerámica al poseer mayor refractariedad que el talco (1). Este hecho hace que diversas publicaciones separen, en la actualidad, a estos dos silicatos (1-9). Se ha llegado a mencionar que incluso la pirofilita llega a ser de menor coste que el talco, a juzgar por algunos datos de la bibliografía (8).

La pirofilita es un silicato mineral más abundante de lo que se pensaba en un principio, existiendo varios yacimientos de importancia distribuidos por todo nuestro planeta. De ellos, los más importantes se encuentran en Japón, Corea del Sur y EE.UU., con más del $90 \%$ de la producción mundial (Tabla I). Algunos datos recogidos en la Tabla I de los que se dispone no son del todo correctos, ya que es problemático saber qué cuantía de los mismos corresponden a pirofilita pura, o bien a mezclas más complejas con otros minerales, además de aparecer conjuntos pirofilita y talco en muchas estadísticas, por ejemplo en Canadá.

En Japón, la producción minera de este grupo de materias primas se reune bajo la denominación "Roseki" $(1,3,8,10)$. En dicho grupo puede encontrarse a pirofilita en un $73 \%$ y caolinita y sericita en un $26 \%$, existiendo también yacimientos de pirofilita denominados "Toseki". Sudo (10) ha realizado una recopilación de los estudios realizados en Japón hasta los años setenta sobre estas importantes materias primas, destacando yacimientos conteniendo pirofilita como es el de Shokozan. Noriyuki (3), en un trabajo posterior, discutió la producción de pirofilita en ese país.

Los yacimientos de pirofilita de Japón se continúan en Corea del Sur, otro importante productor del mineral $(2,8)$. Además de Japón, Corea del Sur es un notable productor de pirofilita, pero generalmente este silicato aparece en forma masiva denominada "agalmatolito" $(2,5)$.

En EE.UU. son conocidos desde antiguo los extensos yacimientos de Carolina del Norte (Guilford, Orange County, Robbins, Kemp, Glendon, Hillsboro, etc.), comercializándose también pirofilita con alto contenido en sericita. En la revisión anual de minerales (7), se ha mencionado que las reservas de pirofilita de Carolina del Norte son superiores a 12 millones de toneladas métricas. Otros yacimientos se encuentran en California y Pensylvania. En Canadá son importantes los yacimientos de Newfoundland $(5,7,8)$.

En América del Sur destacan países como Brasil, Argentina y Perú. Concretamente, en Brasil existen depósitos de agalmatolitos y los importantes yacimientos de pirofilita de Diamantina en Minas Gerais y en Ibitiara (Bahia), denominándose "ibitiarita" a ciertos materiales de mayor contenido en hierro y agalmatolito $(5,11)$.

Países como Australia (yacimiento de Pambula), Nueva Zelanda (Coromandel) y la India (Hamirpur, Bundelkhand), suministran también pirofilita con diversos grados de pureza para el consumo de numerosas industrias y la exportación $(4,5,8,12-14)$.

En Sudáfrica se encuentran materiales conteniendo un $90 \%$ de pirofilita y de elevada dureza (8 en la escala de Mohs), denominados genéricamente "wonderstone", una pirofilita compacta criptocristalina conteniendo rutilo e impurezas carbonáceas, pero otros contienen a veces gran cantidad de caolinita y mica $(1,5,8)$.

Asimismo, ha de indicarse que desde 1976, Turquía ha

TABLA I

ESTIMACIÓN DE LA PRODUCCIÓN DE PIROFILITA EN DIVERSOS PAÍSES. DATOS EN TONELADAS (REFERENCIAS: 1, 2, 5, 8).

\begin{tabular}{|l|l|l|l|l|l|l|l|l||}
\hline \hline País & $\mathbf{1 9 8 0}$ & $\mathbf{1 9 8 1}$ & $\mathbf{1 9 8 3}$ & $\mathbf{1 9 8 4}$ & $\mathbf{1 9 8 5}$ & $\mathbf{1 9 8 6}$ & $\mathbf{1 9 8 7}$ & $\mathbf{1 9 8 8}$ \\
\hline Japón $^{*}$ & $1,620,467$ & $1,025,721$ & 986,294 & $1,414,424$ & $1,355,625$ & $1,270,112$ & $1,241,069$ & $1,266,000$ \\
\hline Corea del Sur & 514,511 & 395,216 & 427,538 & 656,442 & 738,304 & 587,049 & 690,819 & 703,572 \\
\hline EE.UU. & 167,832 & 97,070 & 78,930 & 77,110 & 73,480 & 75,298 & 83,462 & 97,070 \\
\hline Brasil & 82,920 & 180,360 & 46,210 & 56,050 & 85,496 & 92,726 & 48,550 & 178,722 \\
\hline Canadáx $^{x}$ & 91,848 & 82,715 & 97,000 & 122,992 & 126,860 & 123,037 & 136,000 & 150,000 \\
\hline India $^{\text {Australia }}$ & 35,000 & 41,681 & 59,042 & 81,430 & 53,740 & 53,000 & 51,724 & SD \\
\hline Argentina & 11,002 & 8,146 & 9,569 & 7,051 & 7,317 & 8,588 & 6,036 & SD \\
\hline Sudáfrica & 8,156 & 2,478 & 6,312 & 5,012 & 2,785 & 2,812 & 1,260 & SD \\
\hline Otros & 4,900 & 5,662 & 3,575 & 3,851 & 4,227 & 4,606 & 3,467 & 3,162 \\
\hline \hline
\end{tabular}

* A partir de 1983 inclusive se engloba la producción de pirofilita y arcillas con pirofilita.

$\mathbf{x}$ Los datos incluyen la producción de talco y pirofilita

\# Incluídos Perú y Tailandia

$\mathrm{SD}=\mathrm{Sin}$ datos disponibles; $(\mathrm{e})=\mathrm{V}$ alor estim ado 
comenzado a introducirse en el mercado con tres variedades comerciales de pirofilita: masiva, un material arcilloso blando y otro conteniendo sericita $(8,15)$. Se ha mencionado también la presencia de rocas conteniendo pirofilita en Marruecos (16). En general, según los procesos geológicos que conducen a la formación de pirofilita (y en los cuales no se incidirá en la presente revisión), se pueden encontrar en estos yacimientos los siguientes minerales (1-18):

- pirofilita de elevada pureza

- pirofilita conteniendo cuarzo

- pirofilita con alto contenido en alúmina, procedente de diásporo que la acompaña, corindón, caolinita, dickita y nesosilicatos de aluminio (cianita, andalucita, silimanita), además de topacio

- pirofilita conteniendo mica moscovita, interestratificados de diversa naturaleza, feldespatos, óxidos de hierro, rutilo, carbonatos y otras impurezas.

En el caso de existir cuarzo, lo cual viene a ser frecuente, el material será abrasivo. Si se encuentran presentes otros silicatos de aluminio como caolinita, cianita, etc., aumentará el contenido en alúmina y, en consecuencia, la refractariedad será mayor a igualdad de elementos restantes. La presencia de micas y feldespatos elevará el contenido en álcalis, repercutiendo de manera desfavorable en la refractariedad y otras propiedades. Análogamente ocurrirá al existir óxidos de hierro, carbonatos, además de otras especies minerales acompañando a la pirofilita. Por todo ello, en general es preciso realizar un proceso previo de purificación para mejorar la calidad del producto final, destinado a muy diversas aplicaciones como se verá más adelante. Por ejemplo, se han obtenido muy buenos resultados empleando procedimientos de purificación por flotación con aceite de pino (19), surfactantes (20) y molienda diferencial seguida de una separación por flotación con aire (21).

\subsection{Yacimientos de materias primas conteniendo pirofilita en España}

En nuestro país, a fines de la década de los cincuenta Martín Vivaldi y Pino Vázquez (22) identificaron la asociación mineralógica caolinita-mica-pirofilita en arcillas de la localidad de Zarza de Alange (Badajoz). Posteriormente, numerosos estudios ha puesto de manifiesto la existencia de pirofilita, asociada a otros minerales, en yacimientos situados principalmente en la provincia de Badajoz y estudiados en sus trabajos por Alvarez Estrada (23), Alvarez Estrada y Sánchez Conde (24), Mesa et al. (25), Mesa y García Ramos (26), García Ramos et al. (27), Mesa $(28,29)$ y más recientemente Sánchez Soto et al. (30); en Ciudad Real, estudiados por Galán Huertos y Martín Vivaldi (31) y, especialmente, Espinosa de los Monteros et al. (32) y en Córdoba, estudiados por Tirado y Paneque (33), Tirado et al. $(34,35)$ y Bellinfante et al. (36), investigaciones que permitieron además establecer muchas de sus aplicaciones en función de sus propiedades tecnológicas.

Destacan los estudios más sistemáticos realizados por Galán Huertos y Martín Vivaldi $(31,37)$, Galán Huertos y Espinosa de los Monteros (38) y Galán Huertos (39) dentro de las investigaciones sobre la geología, mineralogía y génesis de caolines españoles, así como los llevados a cabo por Mesa $(28,29)$ sobre los importantes yacimientos de pizarras alumínicas, con o sin pirofilita, existentes en la provincia de Badajoz, además de las revisiones debidas tanto a este mismo investigador (29) como a Rincón López (40) sobre materias primas de esta naturaleza en Extremadura publicadas en un trabajo monográfico.

Asimismo, debe mencionarse que se conocen indicios de pirofilita en otras provincias españolas, como son Murcia, según los estudios realizados por Acosta Echevarría (41); en Granada, de acuerdo con Gómez Pugnaire et al. (42); en Málaga, según Moreno Real et al. (43); en Huelva, está bien documentada la presencia de pirofilita conforme a las investigaciones llevadas a cabo en varios trabajos por Maqueda et al. (44), Pérez-Rodríguez y colaboradores (45) y Rodríguez Rubio (46), así como en la provincia de Cáceres, de acuerdo con Sánchez Camazano et al. (47). Estos últimos autores han cuestionado la presencia de pirofilita en la provincia de Málaga (43).

En este sentido, ha de indicarse que aunque talco y pirofilita se asemejan en muchas propiedades, excepto su refractariedad, como ya se ha mencionado (y a veces la identificación por difracción de rayos $\mathrm{X}$ resulta ser también ambigua), el método químico de purificación y aislamiento de pirofilita, puesto a punto por Pérez Rodríguez y colaboradores $(44,45)$ y basado en su alta inercia química en medio ácido fuerte, permite una clara distinción entre ambos silicatos laminares por disolución selectiva en mezclas ácidas.

Como comentario final de este apartado, se sugiere que con un mayor esfuerzo en investigación podrían conocerse las potencias de los yacimientos mencionados, así como la posible purificación o tratamiento de las materias primas ricas en pirofilita, determinarse las propiedades, en particular las tecnológicas, y establecerse las aplicaciones a las que podrían destinarse los materiales, lo que revalorizaría económicamente las zonas donde aquéllos se encuentran.

\section{APLICACIONES PRINCIPALES}

Como se ha mencionado anteriormente, en la literatura antigua se conocía a la pirofilita con los nombres de agalmatolito, piedra imagen, pagodita y "piedra maravillosa" ("wonderstone") $^{\prime \prime}(1,5,8,11)$. Estas ancestrales denominaciones estaban relacionadas con las aplicaciones del material, tales como su uso en la construcción de hornos para trabajar los metales y como material para tallar pequeñas estatuas y figurillas.

En la primera parte de esta revisión ya se describieron con profusión las características propiedades térmicas de este silicato y la formación de las fases de alta temperatura, que son las que confieren su carácter refractario.

Por otra parte, la similitud de la pirofilita con el talco, excepto su refractariedad, hace que se utilice en muchas industrias que consumen este segundo mineral. No obstante, se diferencia del talco en que no suele contener asbesto, aunque sí cuarzo. Por ello, se han realizado algunas investigaciones sobre su repercusión en la salud (48).

En la actualidad, los campos principales de aplicación de la pirofilita son la industria cerámica y de refractarios, como material resistente al calor y en otras aplicaciones como relleno y soporte, así como en la síntesis de nuevos materiales como se describirá a continuación. Esto hace que sea una materia prima de notable interés industrial. No obstante, en la última revisión anual de la Sociedad Americana de Cerámica no aparecen datos estadísticos sobre producción de pirofilita (7).

A título orientativo, en la Tabla II se muestran comparativamente los porcentajes de utilización final de la pirofilita en EE.UU., calculados a partir de los datos disponibles de producción desde 1979 a 1988 para este país, propios de pirofilita $(1,5)$. Se observa que en el transcurso de dicho período 
1979-88 se mantiene el porcentaje de utilización de pirofilita en cerámica, disminuyendo comparativamente su uso en insecticidas, refractarios y construcción, aumentando su porcentaje de aplicación en otros usos (por ejemplo, como carga en plásticos). La principal razón de la disminución de su uso en refractarios (Tabla II) ha sido la baja demanda por dicha industria debido a los cambios realizados en la producción del acero (5), tradicionalmente uno de los mayores consumidores.

\subsection{Materiales refractarios}

La pirofilita es uno de los silicatos de aluminio que presenta un particular interés en la preparación de refractarios por las razones siguientes:

(1) Alta estabilidad a la temperatura por formarse mullita y cristobalita mediante tratamiento térmico.

(2) Baja deformación en caliente, es decir, también posee alta refractariedad bajo carga debido a la formación de fases vítreas muy viscosas, puesto que en el diagrama de fases $\mathrm{SiO}_{2}-\mathrm{Al}_{2} \mathrm{O}_{3}$ la composición cae más cercana a la de sílice pura (Figura 10).

(3) Baja conductividad térmica, lo cual es de interés para la preparación de refractarios aislantes.

(4) Baja expansión térmica reversible, lo cual es importante desde el punto de vista de la resistencia al choque térmico.

(5) Baja densidad, aunque es función de cómo se haya realizado el procesamiento del material.

(6) Alta resistencia a la corrosión por metales fundidos y escorias básicas.

Por ello, se utiliza en la fabricación de crisoles para fundición de metales, entra a formar parte de composiciones refractarias plásticas, mezclas de "gunitado", refractarios para vagonetas de hornos, ladrillos aislantes, chamotas, refractarios monolíticos, etc., así como en ladrillos refractarios sin cocer, mezclada con arcillas plásticas y prensada en seco, resistiendo hasta cerca de $1700^{\circ} \mathrm{C}$ si es de elevada pureza (49). Además, se han patentado mezclas sellantes y masas de relleno refractarias a base de pirofilita, utilizando resinas fenólicas como aglomerantes (50) y cementos refractarios (51).

Siendo Japón el principal productor de pirofilita, se han estudiado sus aplicaciones principalmente por investigadores de dicho país $(3,10)$. Así, por ejemplo, en la industria del acero son muy atractivos los refractarios japoneses por sus buenas propiedades $(3,8)$. En particular, se ha mencionado que ofrecen

TABLA II

UTILIZACIÓN DE PIROFILITA PARA DIVERSOS USOS EN EE.UU. CALCULADOS SOBRE LA BASE DE LOS DATOS DE PRODUCCIÓN 1979-1988 (REFS. 1,5)

\begin{tabular}{|l|l|l|l|l|l|l|l|l||}
\hline \hline UTILZACION & $\mathbf{1 9 7 9}$ & $\mathbf{1 9 8 0}$ & $\mathbf{1 9 8 1}$ & $\mathbf{1 9 8 2}$ & $\mathbf{1 9 8 3}$ & $\mathbf{1 9 8 4}$ & $\mathbf{1 9 8 7}$ & \\
\hline Cerámica & $33 \%$ & $9 \%$ & $11 \%$ & $23 \%$ & $31 \%$ & $61 \%$ & $61 \%$ & \\
\hline Insecticidas & $17 \%$ & $19 \%$ & $27 \%$ & $22 \%$ & $14 \%$ & $10 \%$ & $9 \%$ & \\
\hline Refractarios & $30 \%$ & $48 \%$ & $36 \%$ & $26 \%$ & $28 \%$ & $15 \%$ & $15 \%$ & \\
\hline Construcción & $7 \%$ & $7 \%$ & $8 \%$ & $12 \%$ & $8 \%$ & $4 \%$ & $1 \%$ & \\
\hline Otros usos & $12 \%$ & $16 \%$ & $17 \%$ & $16 \%$ & $18 \%$ & $10 \%$ & $14 \%$ & \\
\hline
\end{tabular}

* Los datos de 1985 y 1986 son sim ilares a los correspondientes a 1984. excelentes prestaciones en las cucharas de colada $(5,52,53)$.

Es de destacar su alto nivel de resistencia al ataque por escorias fundidas y metales debido a la formación de lo que podría denominarse una barrera protectora de material con alta viscosidad originado por interacción en la interfase de escoria y pirofilita. Dicha barrera reduce de manera efectiva la velocidad de destrucción del refractario de pirofilita e incrementa su duración media en condiciones de servicio (5).

A partir de pirofilita se han preparado diversos materiales refractarios con alto contenido en mullita para su empleo en la industria del acero. Jeffries (54) en 1977 obtuvo buenos resultados por reacción entre pirofilita y andalucita. Cabe mencionar también que Pepplinkhouse y Tauber (55) consiguieron preparar refractarios de este tipo por reacción entre pirofilita y gibsita, estudiando la acción de diversos mineralizadores $\left(\mathrm{MgO}, \mathrm{TiO}_{2}\right)$ sobre el proceso. Materiales de gran interés se obtienen a partir de alúmina y pirofilita, siendo útiles para aplicaciones de alto voltaje y sistemas microelectrónicos (56) y como refractarios con alto contenido en alúmina (57). Otros dispositivos refractarios de interés fabricados a partir de pirofilita son los intercambiadores de calor resistentes a los álcalis (58).

Se ha indicado que pirofilita de Australia, con un $14 \%$ de $\mathrm{Al}_{2} \mathrm{O}_{3}$ en peso y un contenido de $\mathrm{K}_{2} \mathrm{O}$ por debajo del $0,5 \%$, se utiliza en refractarios de contacto y una pirofilita con sericita, con un $18 \%$ de $\mathrm{Al}_{2} \mathrm{O}_{3}$ y contenido de $\mathrm{K}_{2} \mathrm{O}$ inferior al 0,7 \%, se utiliza en dispositivos para hornos de tipo túnel (5).

Por otra parte, se conocen métodos patentados para preparar refractarios con pirofilita y carburo de silicio, utilizados en dispositivos de hornos y como aislantes $(59,60)$, así como refractarios especiales conteniendo nitruro de silicio (61) y ácido fosfórico (62).

Cabe destacar que un método bastante común de preparación de refractarios consiste en mezclar la pirofilita con zircón $\left(\mathrm{ZrSiO}_{4}\right)$, originando los refractarios denominados zircón-pirofilita útiles en la fabricación del acero $(5,63,64)$. El zircón es caro, y resiste unos 80 calentamientos en condiciones de servicio. La propia pirofilita sólo llega a unos 30/35. Al enriquecer pirofilita con zircón, se consigue preparar un refractario más económico, lográndose aumentar a 50 calentamientos $(63,64)$. También se utilizan estos refractarios en buzas sumergidas (1)

Asimismo, se pueden preparar otros tipos de refractarios mezclando arena de zircón, pirofilita y aglomerante, prensando el material y utilizándolo directamente sin tratamiento térmico (65). Se ha mencionado también que el zircón también puede aglomerarse añadiendo pirofilita, obteniéndose refractarios dentro del sistema ternario $\mathrm{ZrO}_{2}-\mathrm{Al}_{2} \mathrm{O}_{3}-\mathrm{SiO}_{2}$ que son especialmente útiles en zonas de contacto para vidrio fundido (66). Por ejemplo, en la Figura 1 se muestra la situación de este tipo de materiales refractarios en el diagrama de fases mencionado (66).

Materiales de alta resistencia al choque térmico, como Bespodumeno y cordierita, se logran preparar a partir de pirofilita con procedimientos de obtención patentados $(15,67,68)$.

Otros materiales de interés relevante como la celsiana (silicato de aluminio y bario), se pueden obtener a partir de pirofilita y se aplican como protectores de los rayos X (69). Asimismo, según Erndt (70), la pirofilita se emplea también para prevenir defectos en la manufactura de moldes para colado de precisión de aleaciones de aluminio. 


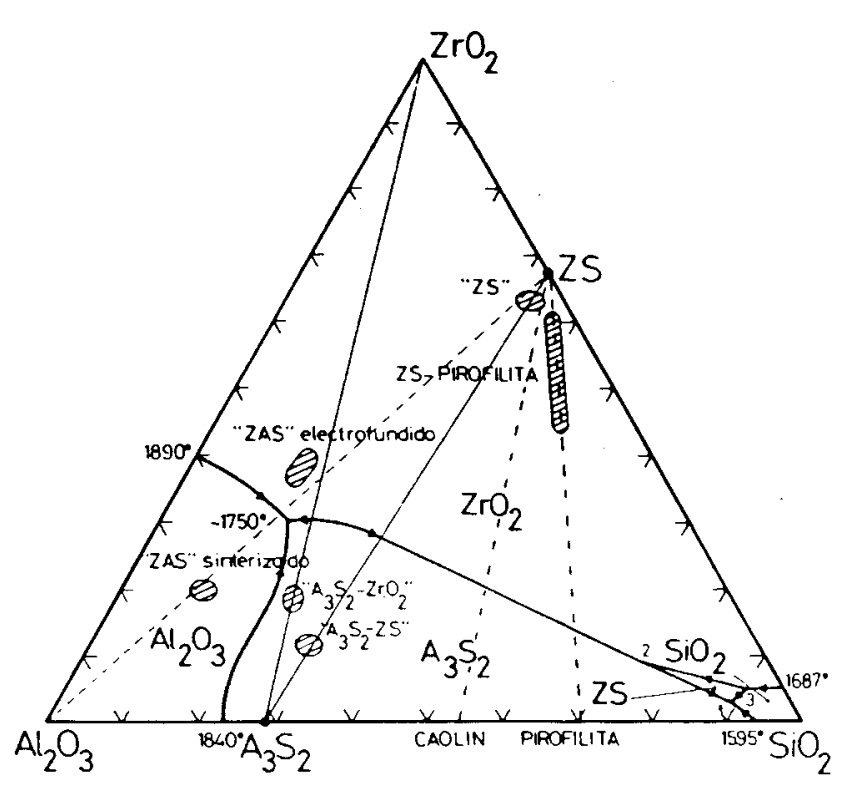

Fig. 1.- Sistema $\mathrm{ZrO}_{2}-\mathrm{Al}_{2} \mathrm{O}_{3}-\mathrm{SiO}_{2}$ (ZAS) con la situación de la composición de diferentes materiales refractarios, según Pena (66).

\subsection{Cerámica}

Aparte de utilizar este silicato en refractarios, destaca por su gran importancia la aplicación del mismo en la industria cerámica, existiendo numerosos estudios y composiciones patentadas en este aspecto. Se pueden mencionar algunos ejemplos notables, como pueden ser su utilización en la preparación de porcelanas especiales (71), materiales cerámicos porosos $(68,72)$ y productos antiácidos para evitar la corrosión en chimeneas (73).

Por sus propiedades aislantes y dieléctricas, entre otras aplicaciones, se ha empleado en la fabricación de aisladores eléctricos conteniendo $94-96 \%$ de pirofilita (49) e incluso para preparar materiales compuestos aislantes empleando polímeros (74).

En particular, ha de mencionarse en la presente revisión que investigadores australianos han publicado varios trabajos sobre propiedades cerámicas y aplicaciones de la pirofilita existente en Australia, principalmente en el yacimiento de Pambula (13). Dichos trabajos han contribuido a un mejor conocimiento y desarrollo de sus posibilidades, dentro de la industria cerámica, como sustituto del cuarzo y materiales silíceos, por ejemplo en la preparación de porcelanas, azulejos, etc. $(55,75-77)$.

Por sus propiedades refractarias, entra a formar parte de algunos vidriados (78) y esmaltes resistentes a altas temperaturas $(14,79)$.

A partir de los trabajos pioneros de Sproat en los años treinta (80) y Emrich en los cuarenta (81), principalmente, la pirofilita con alto contenido en sericita se viene utilizando desde hace tiempo como sustituto del cuarzo y feldespatos en pastas cerámicas, sobre todo en la manufactura de porcelanas. De este modo, se obtienen buenas propiedades en el secado y cocción, mayor resistencia mecánica, aumento del grado de vitrificación, decrecimiento de la temperatura y el tiempo de cocción y reducción del cuarteo y la contracción, siendo una de las aplicaciones cerámicas más ampliamente difundidas. La sericita contribuye a la formación de mullita a baja tempe- ratura, obteniéndose una buena microestructura intergranular y un aumento de la resistencia mecánica. Todo ello repercute de manera favorable en el consumo energético utilizado en el procesamiento $(82,83)$.

En la actualidad, se observa una tendencia al aumento de su consumo por la industria cerámica al cambiar a la cocción rápida ( $<30$ minutos) y a la utilización de productos comerciales (por ejemplo el denominado "PYRAX RG") para recubrir refractarios y moldes en la industria de fundición, ya que la naturaleza laminar de esta pirofilita, en particular, se afirma que produce coladas con una mejora de su superficie, lo que resulta en un costo más bajo en limpieza $(7,82)$.

En este sentido, han de mencionarse también los trabajos de Zaykov y colaboradores (9) sobre materias primas conteniendo pirofilita de Los Urales. Utilizaron muestras de 5 a $15 \mathrm{Kg}$ para estudiar el comportamiento del material a la molienda, flotación y calentamiento, realizando ensayos industriales con muestras desde 1 a 1200 toneladas para la producción de tejas cerámicas, productos antiácidos y materiales resistentes al calor a partir de materias primas de cuarzo-pirofilita y cuarzo-sericita-pirofilita. Las primeras sinterizaron en torno a $1250^{\circ} \mathrm{C}$, mientras que las segundas lo hicieron a $1100^{\circ} \mathrm{C}$. Estos investigadores encontraron que ambos tipos de materias primas son útiles para la preparación de tejas cerámicas, vidriados, materiales cerámicos domésticos y sanitarios y productos resistentes al calor. Con una mezcla de $30 \%$ en peso de cuarzo-sericita-pirofilita y $70 \%$ de arcillas y nefelina, es posible obtener tejas cerámicas con las siguientes características:

contracción $=3,1 \%$; capacidad de absorción de agua $=7,4 \%$; porosidad abierta $=15,1 \%$ en volumen y densidad aparente $=$ $2,1 \mathrm{~g} / \mathrm{cm}^{3}$.

Asimismo, se obtuvieron muestras experimentales para aislamiento térmico con la siguiente composición: corindón $65 \%$ en peso; cuarzo-pirofilita $23 \%$ y fosfato de aluminio-cromo como ligante $12 \%$. Después de calentar a $210{ }^{\circ} \mathrm{C}$, el producto obtenido posee una densidad aparente de $2,1 \mathrm{~g} / \mathrm{cm}^{3}$ y una resistencia bajo presión de $45 \mathrm{MPa}$.

El diagrama de composición química de la Figura 2 muestra los tipos de materias primas conteniendo pirofilita, según Zaykov y Udachin (9b), en relación a otras materias primas con pirofilita en áreas de composición, según la demanda de la industria.

\subsection{Cargas}

La pirofilita, convenientemente granulada, se puede utilizar además como una carga inerte y diluyente en formulaciones de insecticidas y productos químicos y farmaceúticos para su dispersión (84), lubricantes para trabajar en frío metales y aleaciones (85), como relleno en papeles dieléctricos (86) y surfactantes (87). Entra a formar parte de ciertos composites con poliésteres (88), poliestireno (89), materiales reforzados con fibras (90) y en extintores de incendios (91).

Merece destacarse su aplicación como extensor en la industria de lacas y pinturas, ya sea finamente molida o micronizada; en la industria del papel, plásticos y caucho así como en aplicaciones especiales (cosmética, farmacia, etc.). Para papel, se ha estudiado su efecto en comparación con la caolinita, mencionándose que la retención obtenida es más favorable en el caso de utilizar pirofilita $(8,11)$.

Su uso en productos empleados como agroquímicos, biocidas, fertilizantes y en alimentación animal está ampliamente documentado en diversos países productores, como Japón (5). 


\subsection{Construcción}

En la industria de la construcción se emplea como un material de relleno y sellante de juntas, en la fabricación de cementos, construcción de carreteras y de ciertos sectores peligrosos en ingeniería civil, como son los puentes, túneles e intersecciones (5). Las propiedades reflectivas de pirofilita aumentan la visibilidad nocturna y producen una superficie más conveniente que otros materiales convencionales. Este podría ser un importante mercado para pirofilita en países productores.

Investigadores australianos ya mencionados han demostrado las buenas propiedades que pueden conseguirse con materiales conteniendo pirofilita utilizados en la construcción de carreteras (92).

\subsection{Catalizadores}

Se han investigado algunas de sus propiedades como catalizador, mencionándose su aplicación en reacciones de síntesis orgánica, obteniéndose buenos resultados por ejemplo en la transformación del epoxiestireno a fenilacetaldehido según Ruiz-Hitzky y Casal (93). También se ha encontrado cierta actividad catalítica de la pirofilita en la reacción de deshidratación del etanol a $350-550{ }^{\circ} \mathrm{C}(94)$.

Por otra parte, empleando determinados tratamientos, pueden lograrse modificaciones que originan procesos de quimisorción (95) que pueden ser de interés en ciertas reacciones. Asimismo, se puede utilizar como aditivo en la purificación de hidrocarburos (96), adsorción de aceites (97) y descontaminación radiactiva (98).

Se ha estudiado también su efecto de toxicidad sobre las células una vez molida (99) y sobre la actividad metabólica de ciertos microorganismos (100).

\subsection{Dispositivos de alta presión}

La pirofilita se suele emplear, generalmente, en las cámaras de reacción utilizadas en la manufactura de diamantes sintéticos y síntesis de nuevos materiales obtenidos empleando altas presiones. Por ejemplo, la variedad compacta denominada "wonderstone" en Sudáfrica. El material se corta directamente y actúa como medio de transferencia de la presión, manteniendo su estructura. La pirofilita compacta es fácilmente trabajable, relativamente inerte en las reacciones de alta presión y actúa como aislante eléctrico, siendo una de sus aplicaciones más difundidas y de mayor interés desde hace varias décadas $(5,101-103)$.

Cabe destacar que a principios de la década de los sesenta, Giardini et al. (101) demostraron la formación de coesita, una forma de alta presión de la sílice, y cianita a partir de pirofilita sometida a altas temperaturas y presiones elevadas, como se muestra en la Figura 3, donde se aprecia un diamante en el bloque de grafito después del tratamiento a alta presión y temperatura.

\subsection{Nuevos materiales y materiales avanzados}

Mediante reducción carbotérmica de pirofilita, se han preparado materiales cerámicos de altas prestaciones termomecánicas dentro del sistema $\mathrm{Si}-\mathrm{Al}-\mathrm{O}-\mathrm{N}$, destacando las investi-
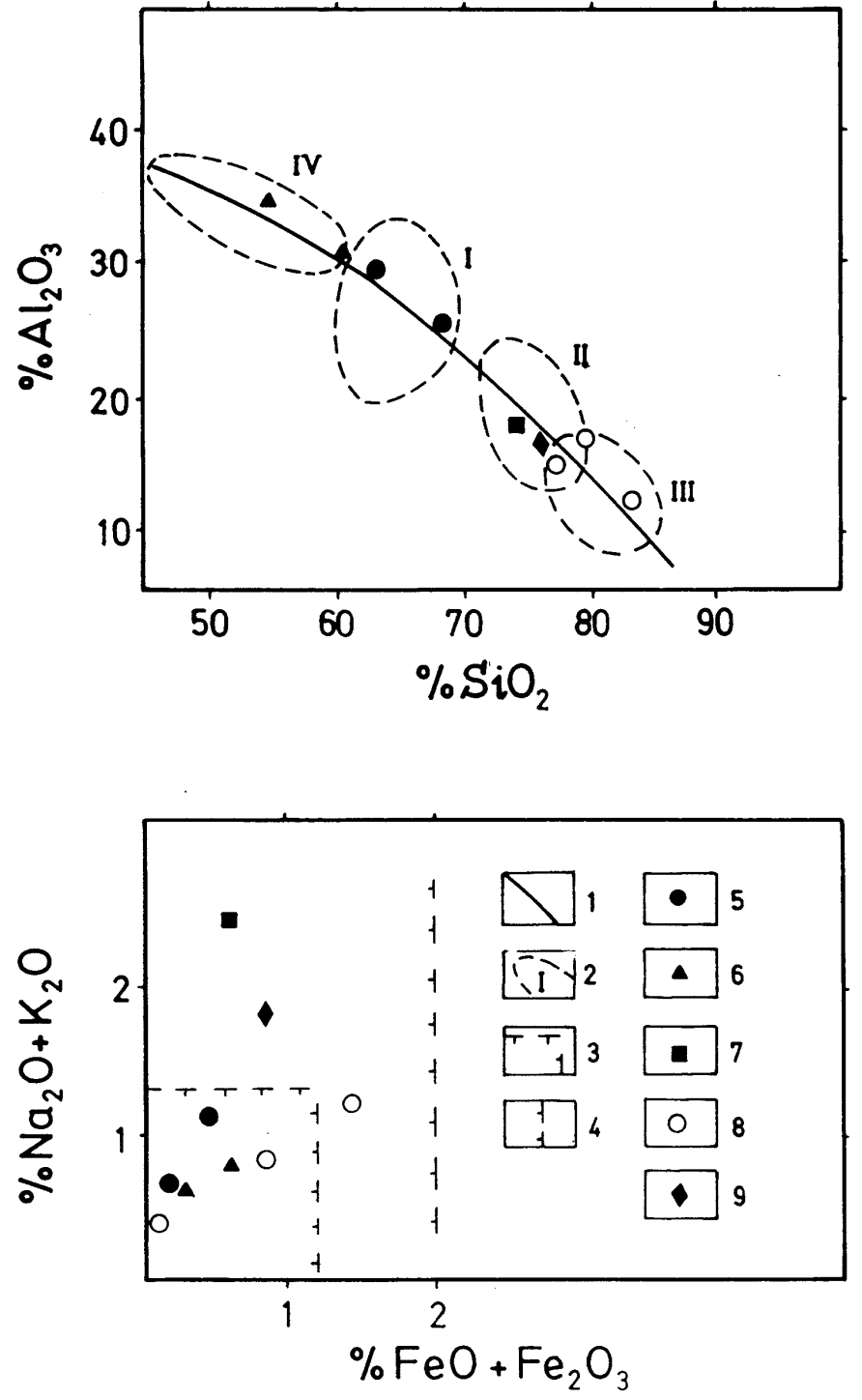

Fig. 2.- Diagramas de composición química de materias primas conteniendo pirofilita en la zona de Los Urales (adaptado de ref. 9): 1 Tendencia de la composición de las materias primas; 2 Límite de zonas de composición en áreas paleovocánicas mundiales con materias primas conteniendo pirofilita (I pirofilítico, II cuarzo-sericita-pirofilítico, III pirofilita-cuarzo, IV pirofilita-diaspórico); 3-4 Límite de zonas de composición de materias primas que demanda la industria: 3 térmicamente resistente, 4 cerámica; 5-9 composición de materiales con pirofilita de Los Urales (antigua URSS), según Zaykov et al. (9): 5 pirofilitico, 6 diásporo-pirofilítico, 7 cuarzo-sericita-pirofilítico, 8 pirofilita-cuarzo, 9 caolinita-pirofilita-cuarzo.

gaciones realizadas por Baldo et al. (104), Lee et al. (105), Lee y Jang (106), Sugahara et al. (107) y Mukherji y Bandyopadhyay (108). Se trata de un hidroxialuminosilicato mucho más silíceo que la caolinita, por lo que la reacción parece ser que transcurre según el esquema siguiente:

$$
\mathrm{Al}_{4} \mathrm{Si}_{4} \mathrm{O}_{10}(\mathrm{OH})_{2}+9 \mathrm{C}+3 \mathrm{~N}_{2}=\mathrm{Si}_{4} \mathrm{Al}_{2} \mathrm{O}_{2} \mathrm{~N}_{6}+9 \mathrm{CO}+\mathrm{H}_{2} \mathrm{O}
$$

Por tratamiento térmico a unos $1320-1420{ }^{\circ} \mathrm{C}$, se producen materiales constituidos por $\beta$-sialón y trazas de otras fases, como $\alpha$-sialón, oxinitruro de silicio, $\beta-S i C$ y $\beta-\mathrm{Si}_{3} \mathrm{~N}_{4}(104-108)$, 

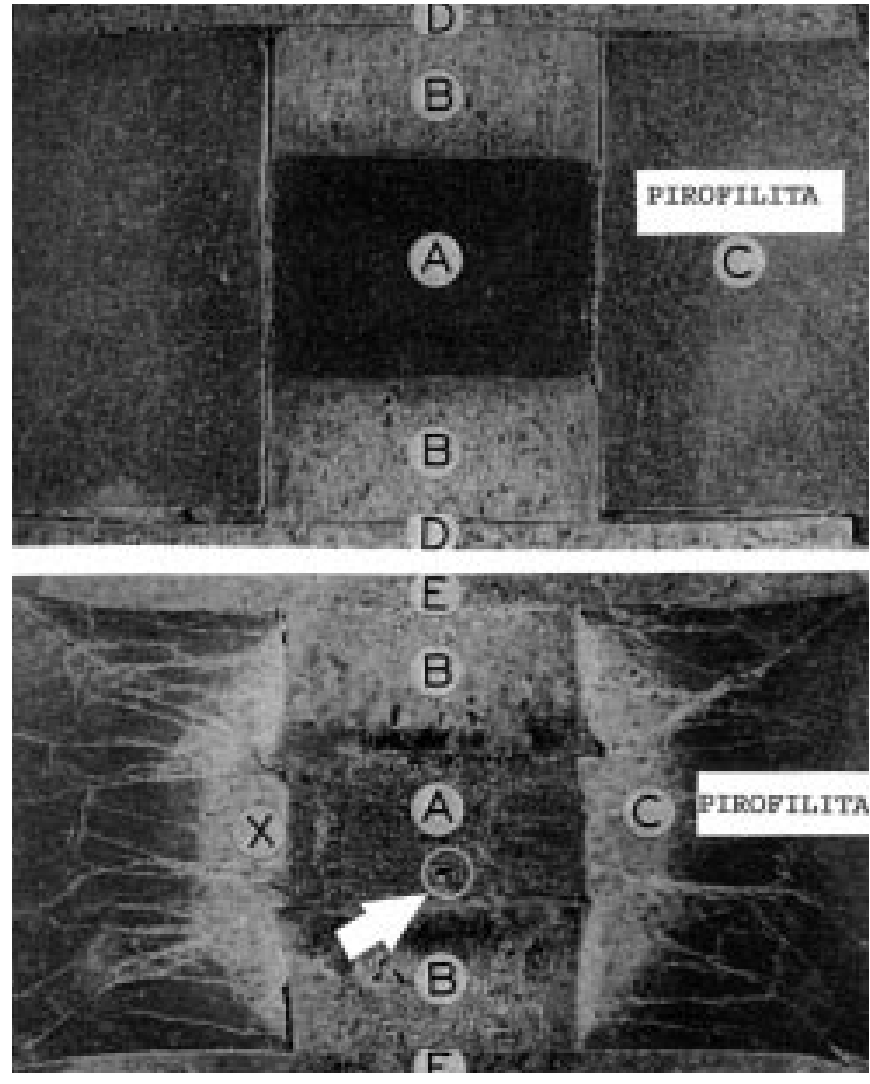

Fig. 3.- a) Sección axial de una cámara de alta presión antes de someter al ensayo: (A) carga de grafito, (B) cilindros de níquel, (C) pirofilita, (D) tapas de níquel de contacto $(x 7,3) ; b)$ Sección axial de la misma cámara después de un ensayo a 85 kbar y calentamiento a aproximadamente $1600{ }^{\circ} \mathrm{C}:(A)$ carga de grafito (se indica la localización de un cristal de diamante sintetizado en este dispositivo), (B) cilindros de níquel (nótese la recristalización), (C) y (X) zona de pirofilita alterada a cianita y coesita, (E) tapas de níquel de contacto ( $x 7,3)$, según Giardini et al. (101).

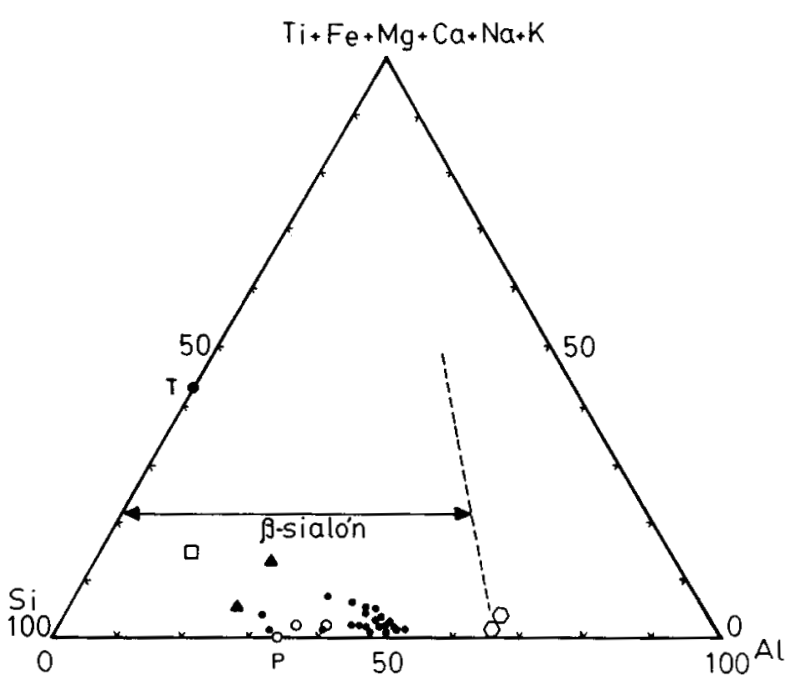

Fig. 4.- Representación gráfica en el diagrama ternario Si-Al$(\mathrm{Ti}+\mathrm{Fe}+\mathrm{Mg}+\mathrm{Ca}+\mathrm{Na}+\mathrm{K})$ de la composición molar para diferentes materiales naturales utilizados en la obtención de polvos de $\beta$-sialón mediante reducción carbotérmica: caolinita y arcillas caoliníticas (círculos negros); arcillas ilíticas y montmorilloníticas (triángulos); materias primas conteniendo pirofilita (círculos vacios); silimanita y materias primas similares (hexágonos); vidrio volcánico (cuadrado). $T=$ Talco; $P=$ Pirofilita. Adaptado de Fabbri y Dondi (109). aunque también pueden existir además mullita y corindón de acuerdo con las investigaciones de diversos autores $(107,108)$.

Según el diagrama de fases $\mathrm{Si}-\mathrm{Al}-(\mathrm{Ti}+\mathrm{Fe}+\mathrm{Mg}+\mathrm{Ca}+\mathrm{Na}+\mathrm{K})$, es posible la obtención de polvos de $\beta$-sialón a partir de pirofilita por reducción carbotérmica y nitruración reactiva (109), como muestra la Figura 4.

Asimismo, se han preparado materiales compuestos a partir de mezclas de carbón y arcillas con pirofilita, procesándolas de forma conveniente (110). Estos nuevos materiales son químicamente estables, porosos y conductores de la electricidad, utilizándose como electrodos de baterías y condensadores. Por otra parte, pirofilita de Diamantina (Brasil) viene siendo utilizada para la producción de ciertas aleaciones de silicio-aluminio (111).

Asimismo, ha de mencionarse que teniendo en cuenta la elevada estabilidad química de pirofilita en medio ácido fuerte, el Grupo de Investigación donde se integran los presentes autores ha realizado diversos estudios para el aprovechamiento de la misma en mezclas naturales con otros silicatos, así como su comportamiento frente a sales que provocan la solubilización del aluminio contenido en estos silicatos, depositándose compuestos de dicho catión sobre el material estable en medio ácido, utilizando para ello el aluminio procedente de los otros silicatos solubilizados. De este modo, se ha logrado la síntesis de mullita a baja temperatura, además de materiales compuestos mullita-alúmina y se continúan las investigaciones en la actualidad (112).

Por último, a modo de resumen, los principales usos de la pirofilita y materias primas que la contienen en gran proporción se muestran en la Figura 5, adaptada y modificada de Robertson (113), donde las aplicaciones se clasifican en función del estado de agregación del material y sus propiedades.

\section{UTILIZACION DE MATERIAS PRIMAS ESPAÑOLAS CONTENIENDO PIROFILITA}

En nuestro país, merece destacarse la continua labor de investigación, plasmada en numerosos trabajos, desarrollada en su mayoría por el Instituto de Cerámica y Vidrio del Consejo Superior de Investigaciones Científicas (C.S.I.C.), el Departamento de Química Inorgánica y el Departamento de Cristalografía, Mineralogía y Química Agrícola de la Facultad de Química de la Universidad de Sevilla, el antiguo Centro de Edafología y Biología Aplicada del Cuarto (C.S.I.C.), hoy Instituto de Recursos Naturales y Agrobiología de Sevilla (IRNAS) del C.S.I.C. y el Instituto de Ciencia de Materiales de Sevilla (ICMSE) Centro Mixto C.S.I.C.-Universidad de Sevilla.

Estos trabajos, realizados desde la década de los sesenta, tanto en su aspecto de prospección de materias primas como desde el punto de vista tecnológico, ya mencionados y citados en apartados anteriores, han servido para demostrar el enorme interés y grandes posibilidades de las denominadas "pizarras alumínicas", con o sin pirofilita, como materias primas en la Industria Cerámica y de formulaciones de insecticidas, caucho, papel, pinturas, etc. y en la síntesis de nuevos materiales.

En particular, con las arcillas conteniendo pirofilita se han logrado preparar porcelanas siliciosas y aluminosas, materiales refractarios de alta alúmina, refractarios medios de baja temperatura de servicio, materiales altamente resistentes al choque térmico (pastas cerámicas de cordierita y cordieritamullita)(32b), productos gresificados, antiácidos, lozas y soportes de azulejos, etc. y se ha probado su adición a pastas cerámicas industriales $(23,24,28,29,32,36,39,40)$. Como ya se 
ha indicado, una revisión de relevancia sobre las aplicaciones de las denominadas "pizarras alumínicas" que existen en la región extremeña, donde este tipo de materias primas se encuadraría, ha sido realizada por Mesa (29) y Rincón (40) en la monografía ya mencionada.

Conviene indicar que el pequeño y homogéneo tamaño de partículas que presentan estas materias primas naturales, junto con la presencia de abundante mica (sericita), entre otros factores, conducen a un aumento en la velocidad de mullitización con un gran ahorro energético $(1,6,7,32,82,83)$. Esto lleva a considerarlas como materias primas de gran importancia. Con un procesamiento idóneo, podrían utilizarse como materias primas para la preparación de nuevos materiales y materiales avanzados, llevándose a cabo diversos esfuerzos para lograrlo.

En particular, nuestro Grupo de Investigación ha finalizado un Proyecto de Investigación dirigido a la "Puesta en valor y nuevas aplicaciones de arcillas que contienen pirofilita mediante tratamientos mecánicos, químicos y térmicos" y financiado por la CICYT, algunos de cuyos resultados y otros previos de interés han sido anteriormente mencionados.

\section{COMENTARIOS FINALES}

A lo largo de la presente revisión (Partes I y II), se han expuesto y descrito las investigaciones realizadas sobre la estructura de pirofilita, sus propiedades más importantes, la síntesis en el laboratorio de dicho hidroxialuminosilicato y la formación de soluciones sólidas. Se ha realizado una revisión de sus características propiedades térmicas en cuanto atañe a los procesos de deshidroxilación y formación de fases de alta temperatura, así como a su rehidroxilación. En algunos de estos aspectos, se mencionan investigaciones realizadas por los autores, destacando aquéllas en relación a la formación de mullita a partir de pirofilita por tratamientos térmicos; en este sentido, además, se describen los resultados obtenidos combinando los tratamientos de activación mecánica por molienda y los térmicos, los cuales resultan ser de gran interés para producir la formación de mullita a temperaturas relativas más bajas en pirofilita y materias primas que la contienen. En particular, también se mencionan algunas investigaciones realizadas aprovechando su alta inercia química en medio ácido fuerte.

Asimismo, se han indicado los principales yacimientos mundiales de este silicato, los datos de producción disponibles y las investigaciones realizadas en nuestro país sobre aspectos de prospección y caracterización de materias primas conteniendo pirofilita, los cuales presentan un marcado interés aplicado. Se sugiere que con un mayor esfuerzo en investigación podrían conocerse las potencias de los yacimientos mencionados, así como la posible purificación o tratamiento de las materias primas ricas en pirofilita, determinarse las propiedades, en particular las tecnológicas, y establecerse las aplicaciones a las que podrían destinarse los materiales, lo que revalorizaría económicamente las zonas donde aquéllos se encuentran.

Como parte importante y de interés, se ha realizado una amplia revisión de las aplicaciones principales a las que se destinan las materias primas conteniendo pirofilita, en especial como materiales refractarios $y$, en general, sus aplicaciones más relevantes en cerámica, su uso como carga, en la construcción, catalizadores y síntesis de materiales a alta presión debido a sus propiedades, resumiéndose esencialmente todo ello en el diagrama de la Figura 5.

Por último, en el presente trabajo, también se han descrito y

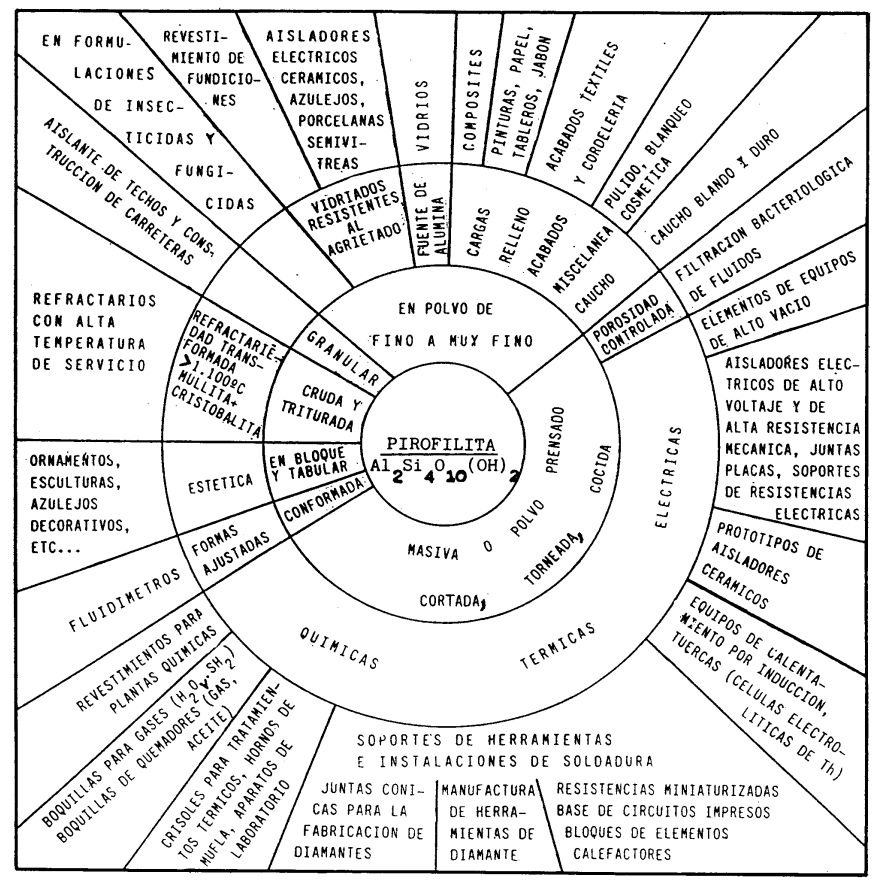

Fig. 5.- Diagrama de utilización general de pirofilita en relación a su estado de división y propiedades, adaptado y modificado de Robertson (113).

revisado sus aplicaciones en la preparación de nuevos materiales y materiales avanzados, así como su utilización en nuestro país como resultado de las investigaciones realizadas por distintos Institutos y Departamentos universitarios, a lo cual los autores del presente trabajo han venido contribuyendo, desde hace tiempo, con la realización de diversos Proyectos de investigación. Estos han dado como resultado, entre otros, diversos trabajos de Tesis de Licenciatura y Doctorado y una serie de artículos científicos de interés, la mayoría ya publicados, sobre este silicato laminar.

De todo lo expuesto con anterioridad, se concluye que la pirofilita va emergiendo cada vez más del conjunto de otros como son talco y caolinita, destacándose como tal con su propia importancia.

\section{AGRADECIMIENTOS}

La presente revisión se ha basado sobre la Tesis Doctoral del primer autor, dirigida por el coautor de este trabajo, dentro de los Proyectos de Investigación financiados por la Junta de Andalucía y C.S.I.C. durante 1985-87 al Grupo de Investigación 6044 "Nuevos materiales a partir de silicatos naturales" de la Junta de Andalucía 1989-1995 y la CICYT (Referencia GE090-0060) durante 1990-93, con el disfrute de una Beca de PFPI del MEC (1986-1989), agradeciéndose a todas estas entidades el apoyo económico que ha hecho posible el desarrollo de las investigaciones realizadas descritas en esta revisión. Asimismo, se agradece también la ayuda económica recibida dentro del Proyecto MAT96-0507. 


\section{BIBLIOGRAFIA}

1. P.W. Harben, Pyrophyllite-stands on its own, Industrial Minerals Refractories Survey 125-129 (1981).

2. K.N. Sang, Pyrophyllite clay deposits of the Republic of Korea, Ind. Miner, 11, 30-31 (1983).

3. F. Noriyuki, The present position of Japanese pyrophyllite, Ind. Miner., 194, 21-27 (1983).

4. D. Nichol, Pyrophyllite operations at Pambula, Australia, Ind. Miner., 194, 3135 (1983).

5. Roskill Information Services Ltd., The Economics of Talc and Pyrophyllite, Sixth Edition, London, 1990

6. IM Indexes, Ind. Miner., Industrial Minerals Information Ltd., Twenty years IM index, Industrial Minerals research, Metal Bulletin, London, 1996.

7. K.C. Rieger, Pyrophyllite-Annual Minerals Review, Am. Ceram. Soc. Bull., 76, 124-125 (1997), The American Ceramic Society Inc., Columbus, Ohio, 1996.

8. B. Ullrich, L. Lesch, Pyrophillit, Silikattechnik, 38, 274-275 (1987).

9. a) V.V. Zaykov, V.N. Udachin, I.V. Sinyakovskaya, Pyrophyllite deposits, Int. Geol. Rev., 30, 90-103 (1988); b) V.V. Zaykov, V.N. Udachin, Pyrophyllite and pyrophyllite raw materials in the sulfide-bearing areas of the Urals, Appl. Clay Sci., 8, 417-435 (1994).

10. T. Sudo, An outline of clays and minerals in Japan, p. 1-103 en: Clays and clay minerals of Japan, Developments in sedimentology No. 26 (Eds. T. Sudo y S. Shimoda), Kodamsha Ltd., Tokyo, Elsevier, Amsterdam, 1978.

11. M. Pimenta, M. Damiani, Agalmatolite-a Brazilian white extender, p. 117-126 en: 8th Ind. Miner. Int. Congr., Boston, 1988.

12. I.C. Loughnan, C.R. Ward, Pyrophyllite-bearing flint clay from the Cambewarra area, N.S. Wales, Clay Miner., 9, 83-95 (1971).

13. E. Tauber, H.J. Pepplinkhouse, Ceramic properties of pyrophyllite from Pambula, New South Wales, J. Aust. Ceram. Soc., 8, 62-64 (1972).

14. M.L. Misra, V.G. Upadhyaya, Use of pyrophyllite from Harmirpur in refractory enamels, Cent. Glass Ceram. Res. Bull., 19, 67-72 (1960).

15. S. Özgen, Ö.L. Cakiroglu, The effect of raw materials on the properties of cordierite bodies, pp. 165-176 en: Ceramics Today-Tomorrow's Ceramics (Editor P. Vincenzini), Elsevier Science Publishers B.V., Amsterdam, 1991.

16. M. Soirat, Argiles d'Afrique du nord et possibilités d'utilisation de la pyrophyllite, L'Ind. Ceram., 698, 599-604 (1976).

17. M.L. Sykes, J.B. Moody, Pyrophyllite and metamorphism in the Carolina slate belt, Am. Miner., 63, 96-108 (1978).

18. P.E. Zalba, Clay deposits of Las Aguilas formation Barker, Buenos Aires province, Argentina, Clays Clay Miner., 27, 433-439 (1979).

19. F.D. Lamb, J. Ruppert, Flotation of a North Carolina pyrophyllite ore, U.S. Bureau of Mines, Report of Investigation No. 4674, 7 p. (1950).

20. E. Sttenberg, P.J. Harris, Surface-chemical and mineralogical properties relevant to the flotation of talc and other layer silicates, Report MINTEK 1985, M209 (Sudáfrica), 22 p. (1985).

21. T. Hayashi, A. Inoue, M. Ogiwara, N. Otsuka, Effect of grinding aid of alkyl silicate on dry refining process of clay minerals, Yogyo Kyokai Shi, 87, 33-42 (1979).

22. J.L. Martin Vivaldi, C. del Pino Vázquez, Estudio mineralógico de una "tierra blanca" de Zarza de Alange (Badajoz), Notas y comuns. Inst. Geol. y Minero de España, 49, 3-20 (1958).

23. D. Alvarez Estrada, Características y aplicaciones técnicas de una sericita española, Rev. Ci. Aplic., 73, 130-136 (1960).

24. D. Alvarez Estrada, C. Sánchez Conde, Características y aplicaciones técnicas de la sericita de El Rosado (Badajoz), Rev. Ci. Aplic., 83, 509-516 (1961).

25. J.M. Mesa, G. García-Ramos, J.L. Pérez-Rodríguez, F. González-García, Occurrence of pyrophyllite in La Serena area, Badajoz, Spain, Int. Clay Conf. Oxford 1978 (Abstract).

26. J.M. Mesa, G. García Ramos, Evaluación industrial de dos yacimientos de arcillas cerámicas de la provincia de Badajoz, VII Convención Nacional de la Industria Química, Sevilla (1980).

27. G. García Ramos, F. González García, P.J. Sánchez Soto, M.T. Ruiz Abrio, Propiedades refractarias y estudio de los productos obtenidos a partir de un conjunto de materiales silicoaluminosos españoles (I), Bol. Soc. Esp. Ceram. Vidr., 24, 67-79 (1985).

28. J.M. Mesa López-Colmenar, Contribución al estudio mineralógico de las pizarras alumínicas (tierras blancas) del Paleozoico de la provincia de Badajoz, Tesis Doctoral, Universidad de Sevilla, 318 pp., 1986.

29. J.M. Mesa López-Colmenar, Materias primas cerámicas de la provincia de Badajoz: Geología, Mineralogía y Aplicaciones, p. 6-31 en: Los materiales cerámicos y vítreos en Extremadura (Ed. J.M0 Rincón López), Edita UNED e Instituto de Cerámica y Vidrio, Mérida, 1988.

30. P.J. Sánchez Soto, A. Justo Erbez, M.C. Jiménez de Haro, J.L. Pérez Rodríguez, M. Raigón Pichardo, J. Pascual Cosp, Caracterización y propiedades cerámicas de una pizarra alumínica que contiene pirofilita, Bol. Soc. esp. Ceram. Vidr., 33, 199-205 (1994).
31. E. Galán Huertos, J.L. Martín Vivaldi, Caolines españoles: Geología, Mineralogía y Génesis. Parte IV. Depósitos Paleozoicos, Neógenos y Cuaternarios, Bol. Soc. Esp. Ceram. Vidr., 13, 523-546 (1974).

32. a) J. Espinosa de los Monteros, D. Alvarez Estrada, F. Morales Poyato, M.A. del Río Sánchez, Arcillas sericíticas, nuevas materias primas cerámicas, Bol. Soc. Esp. Ceram. Vidr., 17, 73-78 (1978); b) J. Espinosa de los Monteros, D. Alvarez Estrada, S. de Aza Pendás, F. Morales Poyato, Pastas de cordierita y de cordierita-mullita obtenidas a partir de arcillas sericíticas, Bol. Soc. Esp. Ceram. Vidr., 18, 149-155 (1979).

33. J.L. Tirado Coello, G. Paneque Guerrero, Composición química y mineralógica de los materiales de un yacimiento de arcilla de Hinojosa del Duque (Córdoba), Bol. Soc. Esp. Ceram. Vidr., 20, 297-301 (1981).

34. J.L. Tirado, G. Paneque, N. Bellinfante, Génesis de pizarras arcillosas en Hinojosa del Duque (Córdoba), Bol. Soc. Esp. Ceram. Vidr., 21, 185-187 (1982).

35. J.L. Tirado, R. Cabanás, C. Espino, G. Paneque, Aspectos morfológicos de la alteración de pizarras arcillosas devónicas de Córdoba (España), Est. Geol., 40, 49-54 (1984).

36. a) N. Bellinfante, J.L. Tirado, G. Paneque, Propiedades técnicas de las arcillas de un yacimiento de Hinojosa del Duque (Córdoba), Bol. Soc. Esp. Ceram. Vidr., 21, 123-125 (1982); b) N. Bellinfante, M. García-Valdecasas, J.L. Tirado, G. Paneque, Ceramic applications of argillaceous materials from Cordoba (Spain), pp. 506-513 en: 5th Meeting of the European Clay Groups 1983 (Euroclay'83), Charles University Press, 1985.

37. E. Galán Huertos, J.L. Martín Vivaldi, Caolines españoles: Geología Mineralogía y Génesis. (I, II y III), Bol. Soc. Esp. Ceram. Vidr., 12, 19-98; 215 228; 333-340 (1973).

38. E. Galán Huertos, J. Espinosa de los Monteros, El caolín en España. Características, identificación y ensayos cerámicos, Editado por la SECV, Madrid, 1974.

39. E. Galán Huertos, Posibilidades de utilización de materias primas españolas en la fabricación de refractarios silicoaluminosos y de alta alúmina, Bol. Soc. Esp. Ceram. Vidr., 20, 33-39 (1981).

40. J.M0 Rincón López, Rocas y minerales de Extremadura como posibles materias primas en la producción de vidrios y materiales cerámicos: una revisión, p. 135-158 en: Los materiales cerámicos y vítreos en Extremadura (Ed. J.M0. Rincón López), Edita UNED e Instituto de Cerámica y Vidrio, Mérida, 1988.

41. A. Acosta Echevarría, Yacimiento de pirofilita de Aguilas, Murcia, Comunicación Privada, 4 p.

42. M.T. Gómez Pugnaire, F.P. Sassi, D. Visona, Sobre la presencia de paragonita y pirofilita en las filitas del complejo Nevado-Filábride en la Sierra de Baza (Cordiller Bética, España), Bol. Geol. Min., 89, 468-474 (1978).

43. L. Moreno Real, A. Rodríguez García, S. Bruque, J. Martínez Jara, Mineralogía de la fracción arcilla de ocho perfiles de suelos de Málaga, An. Edafol. Agrobiol., 42, 405-439 (1983).

44. C. Maqueda, J.L. Pérez Rodríguez, A. Justo, Estudio de pirofilita presente en un suelo Ultic Haploxeralf de la provincia de Huelva, Bol. Soc. Esp. Miner., 9, 107-113 (1986).

45. J.L. Pérez-Rodríguez, C. Maqueda, A. Justo, Mineralogy of soils containing pyrophyllite from Southern Spain: isolation and identification of the mineral, Soil Sci., 150, 671-675 (1990).

46. P. Rodríguez Rubio, Constitución de la fracción arcilla de suelos de la provincia de Huelva, Tesis Doctoral, Universidad de Sevilla, 782 pp., 1990.

47. M. Sánchez Camazano, J. Forteza, L.F. Lorenzo, Occurrence of pyrophyllite in soils from Sierra de San Pedro (Cáceres, Spain), Clay Miner., 23, 339-345 (1988).

48. F.W. Gorbey, P.E. Caplan, W.N. McKinnery, T.C. Cooper, Pyrophyllite dust control in the wall and floor tile industry, Am. Ceram. Soc. Bull., 65, 755-759 (1986).

49. F. Singer, S.S. Singer, Cerámica Industrial Vol. 1, Enciclopedia de Química Industrial (Tomo 9), Ed. Urmo, Barcelona, 1971.

50. Y. Toritani, M. Yoshimura, M. Nakajima, T. Kawakani, Dry refractory sealants, Pat. Jpn. Kokkai Tokkyo 3p 61 40,873 (8640, 873) Cl. CO4B 35/66) Appl. 84/162,079 2 pp, 1986.

51. K. Hara, Cement-based lining composition for steel, Pat. Jpn. Kokai 51,110 (Cl. CO4B) Appl. 73, 99,744 3 pp. (Chem. Abst. 84/P94659 f), 1975.

52. D.H. Hubble, J.T. Shapland, New demands on pouring pit refractories, Proc. Natl. Open Heart Basic Oxygen Steel Conf., 54, 152-161 (1971).

53. J. Coto Alvarez, Refractarios aluminosos, pp. 26-30 en: Curso sobre refractarios: nuevas tecnologías y perspectivas, Oviedo, Abril 1985, Consejería de Industria y Comercio del Principado de Asturias, Oviedo 1985.

54. E.H. Jeffries, Application and results using unfired andalusite-pyrophyllite refractories in pre-bake mode furnaces, Light Met., 383-387 (1977).

55. H.J. Pepplinkhouse, E. Tauber, The formation of mullite from kaolin-gibbsi- 
te and pyrophyllite-gibbsite mixtures, J. Aust. Ceram. Soc., 13, 1-4 (1977).

56. A.K. Kulkarni, D.D. Upadyaya, S.U.K. Rao, Studies on development of alumina substrates, Trans. Indian Ceram. Soc., 41, 25-29 (1982).

57. E.E. Vasil'eva, Conditions for the formation of different crystalline forms of mullite, Steklo Keram., 1, 14-15 (1981).

58. T. Ochiai, K. Shigematsu, K. Matsumoto, H. Ide, Alkali-resistant refractory brick and tube for heat exchanger, Pat. Jpn. Kokai 76,18,711 (Cl. CO4B) Appl. 7489, 4793 pp., 1976.

59. T. Hayashi, H. Kyoden, Y. Namba, Refractory coating for foundry equipment, Pat. Fr. Demande 2, 320, 919 (Cl. CO4B 35/00) Japan Appl. 75/97, 206, 13 pp. (Chem. Abst. 88/ P93221c), 1978.

60. S. Fujimoto, H. Hori, Silicon carbide refractory materials, Pat. Jpn. Kokai Tokyo Koho 79,18,815/Cl CO4 B35/36. Appl. 77/83, 049, 4 pp. 1979.

61. K. Chiba, T. Osaki, H. Moi, Refractory stoppers for blast furnace tapping holes, Pat. Jpn. Kokai Tokkyo Koho 79,11,116 (Cl. CO4B35/36) Appl. 77/76, 894. 1979.

62. A.K. Roy, N.R. Sircar, Torsional creep behavior of phosphate bonded kyanite and diaspore, Cent. Glass Ceram. Res. Inst. Bull., 26, 17-20 (1979).

63. P.W. Wright, Reactions of zircon refractories with molten steel, J. Aust. Ceram. Soc., 20, 47-50 (1984)

64. M. Pauline, R.J. James, Zircon-pyrophyllite: an economical steel ladle refractory, Proc. Natl. Open Heart Basic Oxygen Steel Conf., 62, 246-250 (1979).

65. N. Nameishi, H. Yoshino, S. Uto, Zircon-pyrophyllite unfired refractory bricks, Pat. U.S. 3,752,682 (Cl. 106-57; CO4b) Japan Appl. 70 76,324 7 pp. 1978.

66. P. Pena, Refractarios para zonas de contacto con el vidrio, Bol. Soc. Esp. Ceram. Vidr., 28, 89-96 (1989).

67. S. Udagawa, H. Ikawa, K. Urabe, Formation of $\beta$-espodumene by solid state reaction between pyrophyllite and lithium carbonate, pp. 141-147 en: Proc. Int. Clay Conf. Madrid, 1972.

68. TDK Electric Co. Ltd., Porous cordierite ceramics, Pat. Jpn. Kokkai Tokkyo Koho, JP 58,60,667 (83 60,667) Cl. CO4B 35/16) Appl. 81/159,159 3 pp. 1983.

69. INA SEITO Co. Ltd., Radiation shielding tiles, Pat. Jpn. Kokai Tokkyo Koho JP 59,214,799 (84, 214,799) Cl. G21F1/06 Appl. 83/89,573 2 pp. 1984

70. E.E. Erndt, Materials for manufacturing molds for precision casting, Pat. Ger. Offen 2,715,314 (Cl. B22Cl/00) U.S. Appl. 689,153,24 9 pp. 1977.

71. Y. Isomura, T. Hatano, T. Handa, H. Veno, S. Yasuda, Ceramic molding composition with improved moldability, Pat. Japan Kokai 77, 81, $333 \mathrm{Cl}$. CO4B 19/00. Appl. 75/159, 527, 4 pp. (Chem. Abst. 88/26777a), 1977.

72. K.C. Rieger, Porous ceramic bodies, Pat. U.S. 4, 123, 284 Cl. 106-40R; CO4B 35/14, Appl. 794, 4254 pp., 1978.

73. K. Hara, S. Kageyama, A. Yamamoto, H. Fujita, T. Matsumoto, Water and acid resistant materials for lining chimneys and flues, Pat. Japan Kokai 76,41,729 Cl. CO4B 19/04 Appl. 74/115,409 4 pp., 1976.

74. T. Ichiko, T. Usami, Fillers for electric insulators, Japan Kokai 7567,849 Cl. CO8KL CO4B, 6 Jun 75, Appl. 73 118,777 22 Oct 1973, 4 pp. 1975.

75. a) E. Tauber, H.J. Pepplinkhouse, M.J. Murray, Stoneware bodies based on pyrophyllite, J. Aust. Ceram. Soc., 9, 47-51 (1973); b) E. Tauber, M.J. Murray, H.J. Pepplinkhouse, Earthenware tile body based on pyrophyllite and ortophosphoric acid, J. Aust. Ceram. Soc., 16, 46-49 (1974).

76. a) E. Tauber, H. J. Pepplinkhouse, D.N. Crook, Replacement of silica by pyrophyllite in vitreous china products, Interceram, 25, 195-198 (1976); b) E. Tauber, H. J. Pepplinkhouse, D.N. Crook, Development of a china (porcelain) body from Australian Minerals, Interceram, 26, 211-214 (1977).

77. R. Bowman, E. Tauber, Potential applications of two high grade kaolinitic clays and one pyrophyllite deposit, J. Aust. Ceram. Soc., 15, 5-8 (1979).

78. R. Russell, R. Rowlands, Glaze investigations. I. Effect of various silicas in typical glazes, J. Am. Ceram. Soc., 36, 1-8 (1953).

79. S.F. Mukherji, Importance of pyrophyllite in ceramic industry, Indian Ceram., 26, 123-125 (1983).

80. I.E. Sproat, The use of pyrophyllite in wall-tile bodies, J. Am. Ceram. Soc. 19 135-142 (1936)

81. E.W. Emrich, Use of high sericite pyrophyllite in vitreous bodies, J. Am. Ceram. Soc., 24, 141-144 (1941).

82. K.C. Rieger, What raw materials can do to cut energy consumption?, Ceram. Eng. Sci. Proc., 1, 842-847 (1980).

83. K.C. Rieger, Talc, pyrophyllite and wollastonite, pp. 37-44 en: Proc. Minerals and Chemicals in Glass and Ceramics-The Next Decade (Ed. P.W. Harben), An Industrial Minerals Meeting, Corning, New York, 1981.

84. C. Yakurigaku, K.K. Kenyusho, Pharmaceuticals containing solid acids or solid bases, Pat. Jpn. Kokai Tokyo Koho 81, 92, 215 Cl. A61K35/02 Appl. 79/168, 512. 11 pp., 1981
85. M. Ruehlicke, C. Bischof, H. Kuechler, J. Schlegel, W. Mueller, Lubricant for use in cold-working difficulty formable metals and alloys, Pat. Ger. DD 244,046 Cl. C10M107/00 Appl. 261,365. 4 pp. 1985.

86. A.F. Tishenko, L.I. Kurenmaya, A.A. Duzhak, S.A. Sherstynkova, Elevateddensity paper dielectric containing a heat-stabilizing filler, Bum. Prom-st, 2, 15-17 (1984).

87. T. Akamatsu, T. Yoshikawa, Soil barrier compositions, Pat. Jpn. Kokai Tokyo Koho JP 5908, 781 (8408, 781) Cl. CO9K3/00 Appl. 82/118, 0174 pp. (Chem. Abst. 101/9148r) 1984.

88. R.I. Fabuliak, Effect of filler nature on the molecular mobility and properties of filled maleate polyester, Kompoz. Polim. Mater., 29, 33-36 (1986).

89. R.I. Dryagileva, T.S. Ivaniva, N.A. Kntka, V.T. Rosovitskii, P.V. Daisko, Polymerization of styrene on pyrophyllite as a method for its modification, Plast. Massy, 4, 4-6 (1986).

90. C.A. Erskine, Clay composition, Pat. U.S. 4,118,236 Cl. 106-71 CO4B35/00 Australian Appl. 5, 218. 9 pp. 1978.

91. A.V. Antonov, Powdered fire extinguishing composition, Pat. U.S.R.R. SU 1,012,927 Cl. A62D1/00 Appl. 3,260,039 (Chem. Abst. 99/90507s) 1983.

92. E. Tauber, H.J. Pepplinkhouse, L.W. Middleton, Road surfacing aggregates from ceramic raw materials, J. Aust. Ceram. Soc., 11, 46-50 (1975).

93. E. Ruiz-Hitzky, B. Casal, Epoxide rearrangements on mineral and silica-alumina surfaces, J. Catal., 92, 291-295 (1985).

94. R. Dutta, K.K. Gupta, R.P.S. Jakhar, Catalytic activity of Indian clay minerals, pp. 255-259 en: Proc. Catsympo 80, 5th Natl. Catal. Symp., 1983.

95. I. Vassanyi, S. Szabo, M. Csikos, R. Jelinko, Examination of changes in phase and chemical composition of pyrophyllite-1Tc on chlorination, Hung. J. Ind. Chem., 13, 1-10 (1985).

96. M. Ikematsu, I. Monzyo, K. Sakai, Sorbent deasphalting of asphaltene-containing hydrocarbons Pat. U.S. 4,502,950 Cl. 208-309; 10G21/40 5 p Appl. 82/8,774 13 pp. 1985.

97. A.C. Fortuna, F.A.O. Godron-Zeemann, Lat. Braz. Pedido P.I. 75,02,756 Cl. B01015/06 Appl. 75/2,756 9 pp. 1976.

98. K.K. SYSTEM MAINTENANCE, Cleaning agent for decontamining radiocontaminated surfaces Pat. Jpn. Kokai Tokkyo Koho 3P5871,496 Cl. G21F9/28 Appl. 81/170,347 1983.

99. H. Hayashi, K. Koshi, A. Hamada, H. Sakabe, Structural change of pyrophyllite by grinding and its effect on toxicity of the cell, Clay Sci., 1, 99-108 (1962).

100. H. Morisaki, Effect of solid-liquid interface on metabolic activity of Escherichia Coli, J. Gen. Appl. Microbed., 29, 195-204 (1983).

101. A.A. Giardini, J.A. Kohn, D.W. Eckart, J.E. Tydings, The formation of coesite and kyanite from pyrophyllite at very high pressures and high temperatures, Am. Miner., 46, 976-982 (1961).

102. C.O. Hulse, R.B. Graaf, Effect of temperature on the mechanical properties of solid pressure-transmitting media.II. Pyrophyllite, J. Appl. Phys., 36, 1593-1596 (1965).

103. T.N. Tingle, Retrieval of uncracked single crystals from high pressure in piston-cylinder apparatus, Am. Miner., 73, 1195-1197 (1988).

104. J.B. Baldo, V.C. Pandolfelli, J.R. Casarini, Relevant parameters in the production of $\beta$-sialon from natural raw materials via carbothermal reduction, pp. 437-444 en: Ceramic Powders (Ed. P. Vincenzini), Elsevier, Amsterdam, 1983.

105. H.L. Lee, B.K. Jang, H.B. Lee, Synthesis of $\beta$-sialon from Wando pyrophyllite, J. Kor. Ceram. Soc., 22, 35-42 (1985).

106. H.L. Lee, B.K. Jang, Mechanical properties of $\beta$-sialon prepared from Wando (Korean) pyrophyllite, J. Kor. Ceram. Soc., 23, 17-22 (1986).

107. Y. Sugahara, A. Fukaishi, K. Kuroda, CH. Kato, The carbothermal reduction process of 2:1 type clay minerals, Clay Sci., 7, 29-40 (1987).

108. J. Mukerji, S. Bandyopadhyay, Sialons from natural aluminosilicates, Adv. Ceram. Mater., 3, 369-373 (1988).

109. B. Fabbri, M. Dondi, Clays and other raw materials for the production of sialon ceramic powders, Ind. Ceram., 11, 75-81 (1991).

110. T. Kanbara, T. Yamamoto, H. Ikana, T. Tagana, H. Imai, Porous chemically stable, electrically conducting composites prepared by sintering mixtures of carbon and clay, J. Mater. Sci. Lett., 6, 1195-1197 (1987).

111. O.C. Fursman, L.H. Banning, Experimental smelting of aluminium silicates to produce aluminium-silicon alloys, U.S. Bureau of Mines, Report of Investigation No. 5,575, Washington (1960).

112. P.J. Sánchez Soto, M.C. Jiménez de Haro, A. Justo Erbez, J.L. Pérez Rodríguez, resultados no publicados.

113. R.H.S. Robertson, Industrial uses of clay minerals, Silic. Ind., 38, 33-43 (1973).

Recibido: 9-5-97

Aceptado: 2-4-98 